Please do not remove this page

RMIT

UNIVERSITY

\title{
Application of 3D laser scanner, optical transducers and digital image processing techniques in physical modelling of mining-related strata movement
}

Ghabraie, Behrooz; Ren, Gang; Smith, John; Holden, Lucas

https://researchrepository.rmit.edu.au/esploro/outputs/9921862663201341/filesAndLinks?institution=61RMIT_INST\&index=null

Ghabraie, B., Ren, G., Smith, J., \& Holden, L. (2015). Application of 3D laser scanner, optical transducers and digital image processing techniques in physical modelling of mining-related strata movement. International Journal of Rock Mechanics and Mining Sciences, 80, 219-230.

https://doi.org/10.1016/j.jirmms.2015.09.025

Document Version: Accepted Manuscript

Published Version: https://doi.org/10.1016/j.jirmms.2015.09.025 
Thank you for downloading this document from the RMIT Research Repository.

The RMIT Research Repository is an open access database showcasing the research outputs of RMIT University researchers.

RMIT Research Repository: http://researchbank.rmit.edu.au/

\section{Citation:}

Ghabraie, B, Ren, G, Smith, J and Holden, L 2015, 'Application of 3D laser scanner, optical transducers and digital image processing techniques in physical modelling of mining-related strata movement', International Journal of Rock Mechanics and Mining Sciences, vol. 80, pp. 219-230.

See this record in the RMIT Research Repository at:

https://researchbank.rmit.edu.au/view/rmit:33806

Version: Accepted Manuscript

Copyright Statement: (c) 2015 Elsevier Ltd. All right reserved Creative Commons Attribution 4.0 International License.

Link to Published Version:

http://dx.doi.org/10.1016/j.ijrmms.2015.09.025

\section{PLEASE DO NOT REMOVE THIS PAGE}


Elsevier Editorial System(tm) for

International Journal of Rock Mechanics and Mining Sciences

Manuscript Draft

Manuscript Number: I JRMMS-D-15-00241R1

Title: Application of 3D laser scanner, optical transducers and digital image processing techniques in physical modelling of mining-related strata movement with special focus on mining-induced subsidence

Article Type: Technical note

Keywords: Physical modelling of mining-related problems; mining-induced subsidence; substrata movement; cavity growth mapping

Corresponding Author: Mr. Behrooz Ghabraie,

Corresponding Author's Institution: RMIT University

First Author: Behrooz Ghabraie

Order of Authors: Behrooz Ghabraie; Gang Ren; John Smith; Lucas Holden 


\section{Detailed Response to Reviewers}

\section{Abstract:}

Authors would like to appreciate invaluable comments made by the reviewers. These comments, certainly, have improved the quality of the paper.

In general, authors reduced the number of figures in the manuscript to be suitable for the form of a Technical Note. The data already presented in the reference [16] is abstracted, few sections are deleted and the manuscript is shortened. All the comments by the reviewers are responded and appropriate amendments are applied to address the comments.

Authors hope that the amended version of manuscript would be suitable for publication in the form of a Technical Note.

Following is the detailed response to every comments of the reviewers:

Reviewer \#1:

The core content of this manuscript is the introduction of physical simulation experiments including three kinds of deformation measurement techniques. Authors recall the principles of physical modelling and they provide a brief description of data collection and analysis method together with some application examples. The advantages and disadvantages of the different measuring techniques are also presented. The application of TLS to derive subsidence values and to study crack propagation is presented in more detail. This is a relatively new method in this application to physical modelling.

Whereas digital photography (Photogrammetry) and DIC (3.2 - 3.3) are relatively mature technologies, already applied in physical modelling; optoNCDT and TLS (3.4-3.5) represent already available technologies, whose application to physical modelling is interesting. Therefore, the presented approach has some value, particularly considering the comprehensive application of all method for comparison purposes. However, the approach cannot be considered innovative, since the applied techniques have already been developed by others. The proposal of hybrid TLS-DIC method is a new idea, but it seems just a simple combination of the two methods; in addition, the introduction of technology, photogrammetry and scanning equipment for specific parameters in this experiment (such as resolution and measuring precision) needs further explanation.

In conclusion, the paper summarizes the features of the combined application of available measuring techniques to physical modelling. Though interesting, the results of the paper are not outstanding.

Authors' response:

Authors would like to appreciate the reviewer for the invaluable comments. Following considerations are given with regards to the reviewer's comments.

Authors agree with the reviewer's comment about the hybrid TLS-DIC method. This method is a combination of the two methods. However, the authors intended to propose this method as an alternative, fast performed way to illustrate the results of the physical model that can also be used for cross-checking the results of TLS and DIC methods. 
Further explanation is given for the equipment used in the paper. Table 3 is added. This table summarises some of the key specification of measuring devices.

Reviewer \#2:

This manuscript presents an interesting study on physical modeling of the mining-related problems by making use of optical and laser based measurement technologies. In overall, the content is interesting and attractive. The English is good. However, there are a number of unclear topics, which should be amended before the paper can be published. A major revision is suggested before the final acceptance. More specifically:

Authors' response: Authors appreciate the detailed comments made by the reviewer. Please find the authors' responds to the comments bellow.

Section 1 and 2:

Novelty of the paper, including the physical modeling protocol, the optical and laser based measurement techniques and the data/image analysis algorithms as compared to the existing ones should be better presented.

Authors' response: Comment noted - Sections 1.2 and 4 are revised thoroughly to better present the novelty of the paper. In addition, major revisions are applied to the introduction of monitoring devices to better explain the analytical algorithm and data analysing method. Moreover, suitable references are added that readers can refer to in case detailed explanation of the devices and their working mechanism are needed.

\section{Section 3}

$\lambda \quad$ Fig. 1 and 2 should be better explained for the following questions, i.e. 1) does the 3D TLS (PC \#3) detects only the left part of the model and digital camera (PC\#2) detects the right on the front surface of the model, as shown in Fig. 2?, if not, the exact imaging areas should be indicated with the pink triangles in the renewed Fig. 2; 2) does the optoNCDT (PC\#1) only detects on the top surface?. More detailed description is required for the experimental setup and monitoring devices, otherwise, the readers should guess.

Authors' response: Comments noted, Figures 1 and 2 are edited and further explanation about the location of devices is given in Section 3.

$\lambda \quad$ More details about the monitoring devices should be given, such as the spatial resolution, imaging area, accuracy, sampling time, and the concerning technical specifications should be given.

Authors' response: Comment noted - Table 3 is added that includes necessary information, precision and specification of the measuring devices. Other necessary information is presented in the introduction for each device. 
$\lambda \quad$ The novelty of the protocol of the paper, such as the TLS, optoNCDT, DIC, the imaging analysis algorithm, as compared to the existing ones, should be stressed.

Authors' response: Sections 1.2 and 4 are edited to better explain the novelty of the paper to highlight the benefits of using the newer technologies compared to the existing ones.

$\lambda \quad$ 3.1. Case studies: the methods and procedures for performing the physical modeling of the multiple-seam subsidence should be briefed including Example 1 in [16] and Example 2; what is the boundary conditions when conducted the Examples 1 and 2?; descriptions on how to perform the measurement using the said optical and laser based monitoring techniques should be given.

Authors' response: Section 2.2 is added to further explain the physical modelling construction procedure. The boundary conditions of the Examples 1 and 2 are explained in Section 2.3 and shown in Fig. 1. The method to perform measurement by optical and laser based monitoring techniques are explained in Sections 3.4 and 3.5 .

$\lambda \quad 3.2$. Photogrammetry: how many tracking points are placed on the target surface?; what does it mean by "a sufficient number ...."?; which part of the surface of the model is the target surface?;

Authors' response: Added "one point in every $5 \mathrm{~cm}$ " to make it clear how many tracking numbers are used. As explained in Section 3, the whole front surface of the sample is monitored and photographs of this surface are taken and compared by photogrammetry analysis.

for a specific test case, an analytical formulation should be given for better understanding Fig. 4.

Authors' response: Comment noted - Authors believe that the analytical formulation for performing photogrammetry is very simple and includes basic knowledge of math. The introduction of photogrammetry method is edited in Section 3.2 to explain this method better and provide necessary information for the readers.

$\lambda \quad$ 3.2.1. the algorithms introduced is too simple, more detailed introduction is suggested.

Authors' response: After reviewing the other reviewers' comments it is decided to omit Fig. 5 and briefly explain the simple concept of extracting vertical and horizontal displacement from photographs in Sections 3.2 and 3.2.1.

$\lambda \quad$ 3.3. Digital Image Correlation (DIC): the algorithm of DIC should be given.

Authors' response: Section 3.3 is edited and appropriate references are added that readers can refer to in case more detailed algorithms are needed. Authors believe 
that more analytical description of the method is considered out of the scope of this work.

$\lambda \quad$ 3.3.1. Data acquisition and interpretation: content in this subsection has nothing to do with the "Data acquisition and interpretation".

Authors' response: Comment noted - the names of these sections are amended to better reflect the content.

$\lambda \quad$ 3.3.2. Examples DIC analysis results: the comparison between Fig. 11, 12, 8 and 9 should be quantitative, meanwhile, the physics of the physical simulation should be given to help understand the advancement that the authors were obtained.

Authors' response: Quantitative comparison of the Figures are added to this section and the physics of the phenomenon is further explained.

In overall, the contents in the else sections have the similar problems to the aforementioned and the revisions are strongly suggested on the following aspects:

$\lambda \quad$ The physics of the simulation of multi-seam mining-induced subsidence should be introduced including the model implementation, boundary condition, loading history, testing procedures, testing results, etc.;

Authors' response: Comment noted - an explanation is added in Section 2.2. However, these contents have been thoroughly covered in the reference [16] by the same authors. As the associate editor suggested, it is avoided to repeat the contents of [16] in this paper. Necessary information about the model implementation, boundary condition, loading history, material mixtures and model construction is presented in the Section 2.2 (newly added) and Section 2.3. Readers can refer to the reference [16] for more detailed information.

$\lambda \quad$ With regard to the optical and laser based measurement technologies, besides the schematic illustration of the methods, analytical formulation of the algorithms should be given;

Authors' response: Analytical formulation of the optical and laser based measurement technologies has been added to the appropriate sections. In addition, number of comprehensive references is included for readers to refer in case more detailed formulation is required.

$\lambda \quad$ analysis of the testing results should be quantitative.

Authors' response: Quantitative analysis of the test results is added to Sections 3.2 and 3.3 .

6. Conclusions: 
$\lambda \quad$ it seems that many of the points that the authors claimed are not achieved by this manuscript itself; 2) what are the "recent developments in laser scanning devices and optical transducers"? what are the "new possibilities..."?

$\lambda \quad$ it is strongly suggested rewriting the Conclusions to highlight the finding of the paper with a specific manner.

Authors' response: Comments noted - the whole conclusion section is re-written to address the reviewers' comments and better represent the contents covered in the paper.

Associate editor:

In this manuscript, the authors present the application of a number of more or less new measuring techniques to traditional physical modelling of coal long-walls for subsidence analysis and prediction purposes.

The application of these techniques, already presented by the authors in ref [16] of their paper, though not intrinsically new, represent and advance towards a better interpretation of results of expensive and time consuming physical models.

Both reviewers, traditional users of physical modelling, thinks that the presented application is interesting, even if it does not contain new developments, but the application of new available techniques to a traditional approach.

Based on reviewers' comment and in its own reading of the paper, this editor thinks that a revised shortened version of the paper, where the information already presented in ref [16] is abstracted could provide the basis for a suitable Technical Note.

Therefore, this editor invites the authors to submit a revised version of their manuscript in the form of a Technical Note (removing abstract and limiting the number of figures, if possible), where the main advantages of the combined approach are highlighted and where the information overlapping with a similar previous publication [16] was limited. Additionally, the authors should answer the comments of the reviewers in a separate letter, incorporating the most relevant issues in the new version of the manuscript.

If the authors are willing to submit such a document, it will be considered for publication in the form of a Technical Note.

Authors' response:

Comments by the associate editor are highly appreciated. Authors are willing to submit the revised paper in the form of a Technical Note. Thus, the paper is shortened and the information already presented in the reference [16] is abstracted and repetition is avoided. Abstract is removed and the number of Figures is reduced to be appropriate for a Technical 
Note (12 Figures). However, authors preferred not to delete some Figures as they contain key information, thus, they are grouped with other related Figures as subfigures. In addition, Section 1.1 (Common physical test set-ups) is omitted and a summary of common test setups is presented in Table 1.

All the comments provided by the other two reviewers are responded separately. Based on these comments, mainly, the novelty of the paper in the revised form is stressed and more emphasis is made on the newly introduced methods of optical and laser based measurement techniques. Advantages of the combined approach are also highlighted and the physical modelling protocol is explained better. 
- Application of 3D laser scanner, optoNCDT devices, DIC analysis and photogrammetry in physical modelling of subsidence has been demonstrated

- A new method for monitoring fracture propagation pattern has been introduced

- A physical testing protocol for modelling mining-related problems has been presented 


\title{
Application of 3D laser scanner, optical transducers and digital image processing techniques in physical modelling of mining-related strata movement with special focus on mining-induced subsidence
}

\author{
Behrooz Ghabraie $^{\mathrm{a}, *}$, Gang Ren ${ }^{\text {, }}$, John Smith ${ }^{\text {, }}$, Lucas Holden ${ }^{\mathrm{b}}$ \\ a School of Civil, Environment and Chemical Engineering, RMIT University, Melbourne, Australia \\ ${ }^{\mathrm{b}}$ School of Mathematical and Geospatial Science, RMIT University, Melbourne, Australia \\ * Corresponding author. E-mail address: behrooz.ghabraie@rmit.edu.au (B. Ghabraie).
}

Keywords: Physical modelling of mining-related problems, mining-induced subsidence, substrata movement, cavity growth mapping

\section{Introduction}

Physical and numerical modelling techniques are the primary tools used for investigating geotechnical and mining-related issues [1]. With advances in computer technologies over the past few decades, numerical modelling techniques have become more popular, comprehensive and reliable. Numerical methods are also generally more economical and quicker than physical modelling techniques. However, numerical methods are known to be inadequate in simulating detailed ground behaviour [1], such as strata response and failure around multiple excavations. Physical modelling, on the other hand, continues to be of great interest amongst researchers for investigating complicated mining problems. Physical modelling, at its best, illustrates the physics of the phenomenon, indicates the main principles that cause the most important observations and has the ability to predict behaviour under certain conditions, which may have not yet been known [2]. There are many examples of the successful application of physical modelling in investigating a broad range of mining-related problems [1, 3-7]. In rock mechanics and mining science, physical models allow researchers to observe the actual process of cracking, material failures and development of the ground movement around a model underground excavation [8]. This ability places physical modelling techniques ahead of other methods in investigating the mechanisms at work where the processes of deformation are poorly understood.

One example of the application of physical modelling in developing an understanding of the mechanism of a mining-related problem is mining-induced ground surface subsidence. During the 1970s and 1980s, with the growing problem of ground subsidence, in-depth investigations of subsidence mechanism were conducted by number of researchers, [8-10] for example. Physical modelling was shown to be a particularly useful tool for understanding the mechanism of the substrata movement, crack propagation, caving process and the progressive propagation of the subsidence towards the ground surface [8]. The understanding of the ground movement mechanism, which the physical models provided, played a significant role in developing prediction methods for the mining-induced subsidence at the time that sophisticated numerical models and computer programs were not available.

An increasing demand for energy resources has made multiple-seam coal mining common practice for coal mining operators in countries such as Australia, China and US, [11-13] for example. This has presented new challenges to subsidence engineers, as observational data shows significant change in the magnitude and profile of the subsidence due to multiple-seam mining [14-16]. This problem, together with increased public awareness of the potential dangers of ground subsidence, including safety concerns for the nearby residential towns/structures and damages to the environment (e.g. farmland loss, valley closure, surface cracks, etc.), have required subsidence engineers to better understand the in-depth mechanism behind the multiple-seam subsidence [12, 16-18]. Accurate 
numerical modelling of such complicated problem, which must be able to simulate anisotropic material, non-linear material behaviour, weak interfaces, fracture development, rock caving and large displacement, is deemed to be extremely costly and time consuming. Whereas, physical modelling can, again, play an important role in assisting engineers to understand the mechanisms and ground movement characteristics of multiple-seam mining-induced subsidence.

Many researchers have established their own methods and choice of materials for physical modelling. As a result many different methods of analysis and data acquisition have also been used. Depending on the purpose of the study, photogrammetry, displacement sensors, strain gauges or dial gauges were commonly used to record the required output. A summary of the model materials and measurement systems is presented in Table 1.

\subsection{Improved data acquisition and measurement tools}

Although numerical modelling techniques have undergone significant improvements since they were invented a few decades ago, physical modelling techniques and the monitoring devices in mining-related studies have not been significantly improved from the early models (Table 1). Only recently, a few examples of utilizing newer technologies, such as laser based measuring devices, infrared and acoustic measurement have been reported in the literature [16, 21-23]. It is now considered advantageous to employ some of these new measurement techniques and provide researchers with a practical and yet easy to use systematic approach for putting together the best possible combination of new and old measuring technologies for physical modelling of mining related problems.

In many mining-related problems, studying the mechanism of ground movement due to excavating an underground opening requires understanding the overall ground movement pattern, monitoring fracture growth after multiple extractions, precise measurement of ground deformation and mapping of both horizontal and vertical displacement. The common measurement techniques in the available examples of physical modelling (Table 1), such as stress/strain gauges and photogrammetry, provide researchers with discrete or point-based measurements. Although accurate measurement of the substrata and surface deformation is possible by photogrammetry (by placing sufficient number of target points on the model test), producing a contour deformation map of the whole model is typically deemed to be time consuming with high operational cost. In addition, most of the newly developed methods of crack propagation monitoring, such as, infrared and acoustic measurement, are either expensive to use and computationally taxing or unable to produce an illustrative output.

The recent developments in digital photography and laser based technologies have made it possible to monitor the physical modelling results relatively easier with higher accuracy. Digital Image Correlation (DIC) technique is able to produce a 2D full-field map of the substrata displacement using high resolution photographs. This technique can be a suitable substitute for photogrammetry if a contour map of the substrata displacement is required. In addition, non-contact laser based technologies, such as 3D laser scanners, devices that can measure length, temperature and acceleration without making any contact with the sample (optical displacement transducers, for example) have been improved tremendously over the past decade and became available to research organizations. Utilizing these advanced monitoring devices, image processing techniques and surveying technologies can help researchers measure the dynamic displacement of a point, ground subsidence on a surface and crack propagation pattern around an underground excavation. Measurements from these devices can also be edited by readily available computer programs to generate outputs suitable for further analysis and investigation. 
In this paper, practical application of new optical and laser based technologies are discussed, together with 2D displacement analysing techniques, such as, photogrammetry and image processing. Application of Digital Image Correlation (DIC) analysis to extract 2D full-field displacement measurement, a novel approach of cavity growth mapping by means of 3D Terrestrial Laser Scanner (TLS), subsidence mapping of a surface and real-time one dimensional displacement measurement by means of optical Non-contact Displacement Transducer (optoNCDT) are explained. This paper, for the first time, combines the latest technologies in 3D laser scanning and optical noncontact measuring devices to study two case studies of multiple-seam mining-induced ground surface subsidence. It is believed that deploying these new measuring devices alongside innovative analysis and illustration techniques would significantly improve the quality of the outputs and researchers' interpretation of the results. It would as well reduce the computational/operational cost, time and effort and help eliminating errors which might take place during data acquisition process.

In addition, in the following sections, a practical physical modelling protocol has been introduced based on the purpose of a study, the ability of various monitoring devices to produce different output parameters and other important factors, such as, processing time, accuracy of the measurement and discrete/full-field measurement. This protocol assists researchers to choose the preferred combination of monitoring devices and post-analysing techniques to extract the desired parameters from the physical modelling. It is hoped that the presented modelling protocol could be readily adapted to investigate a range of mining-related and tunnelling problems.

\section{Physical modelling of mining-induced ground surface subsidence}

The most important factor in physical modelling is choosing an appropriate construction material that can simulate and behave in a similar way to the real case problem. The material is a personal choice of the researchers and can vary depending on the purpose of the study, availability and cost of the material. However, the strength of the material, dimensions of the model and density of the constructed material should satisfy the basic conditions in accordance with similarity theory. Under this condition only, a problem can be simulated accurately and measurements can be compared with the real case.

\subsection{Similarity theory}

A scaled physical model has to be designed in accordance with the laws of similarity theory [5]. Whittaker and Reddish [8] stated that for the purpose of physical simulation, only parameters with major influence on the mechanical behaviour of the material should be considered in the calculations, including the strength of the material, its density and geometry. These parameters should satisfy the fundamental condition of the similarity theory as [5]:

$$
\frac{C_{\sigma}}{C_{\rho} \times C_{L}}=1 \quad \text { Eq. } 1
$$

where

$$
C_{L}=L_{p} / L_{m}, C_{\sigma}=\sigma_{p} / \sigma_{m}, C_{\rho}=\rho_{p} / \rho_{m} \quad \text { Eq. } 2
$$

In Eq. 1 and 2, $C_{L}$, is the constant of geometry, $C_{\sigma}$, is the constant of strength, $C_{\rho}$, is the constant of density similarity between the model and prototype case, $L$, is length, $\sigma$ is compressive strength, $\rho$ is density, subscript $p$ stands for prototype and subscript $m$ stands for model.

\subsection{Physical modelling material and construction procedure}


The model construction process, choice of material and strength parameters of the model as well as their relation to the real case problem have been previously discussed by Ghabraie et al. [16]. The same modelling techniques, material mixture ratio and similarity constants are used in the present study. The model is gravity loaded in which rock layers are simulated with sand-plaster-water mixtures and are casted into layers with a thin layer of fine sawdust in between each layer to model stratified rock formation. Wooden blocks are used to simulate the longwall panels. Withdrawing the wooden blocks mimics extraction of longwall panels. The similarity constants and material properties used in this paper are summarised in Table 2.

\subsection{Boundary conditions and plane strain assumption}

While the ratio between the dimensions of the model and prototype case is controlled by similarity theory, the total size of the model should be chosen with respect to the boundary conditions. Boundaries should be placed in a distance from the panels that result in negligible stress fluctuation on the boundaries $[1,6]$. Lubricating the boundaries reduces the friction and results in boundary condition as shown in Fig. 1a. Under this condition, a section of the longwall panel can be assessed under plane strain assumption. This assumption is only valid if one dimension of the longwall panel (length) is sufficiently long such that the effect of the start and end of the panel has negligible influence on the deformation characteristics and stress fluctuation of the middle of the panel. This is commonly the case for coal longwall panels, where a section of the panel along its width can be modelled under plane strain condition.

It should be noted that in order to build the physical model, the sample needs to be casted between front and back supports. Enough lubrication should be applied on these parts to reduce building up any unnecessary stress during casting. It is also assumed that the model thickness has negligible effect on stress fluctuation along its thickness during the test.

\section{Model test set-up and measurement techniques}

The proposed physical modelling set-up encompasses some of the recent technologies (Sec 1.2) for surface and substrata movement analysis. The equipment used in this new model test set-up includes optical Non-contact Displacement Transducer (optoNCDT), Terrestrial Laser Scanner (TLS) and digital camera. Technical specification and measurement precision of these devices are abstracted in Table 3. Each of these devices can be employed for distinct purposes. The output of each method can as well be used to cross check and validate the measurements of other methods. In all of these methods, displacement analyses can be done by monitoring the changes between the initial stage and desired stage of the test. A schematic view of the model test set-up is shown in Figs. 1a and b. The digital camera (in the centre) and TLS (next to the camera) are placed in front of the model to monitor the front surface. The optoNCDTs are placed above the top surface of the model to monitor the surface subsidence. In the following sections, various techniques for data acquisition and interpretation are presented. The application of each method is then discussed by means of two example case studies.

\subsection{Case studies}

To illustrate the application of the proposed physical modelling set-up and measurement techniques, two examples of multiple-seam subsidence problems have been modelled. The two scenarios have different mining configurations (Fig. 1c and d) and were initially designed to improve the understanding of mechanism of the multiple-seam subsidence. The mechanism of subsidence and ground movement development for Example 1 has 
previously been discussed in Ghabraie et al. [16]. This example is a model of two partially overlapping longwall panels with thin interburden thickness (Fig. 1c). The upper panel was extracted first (stage 1 ) and after the model was stabilized, the lower panel was extracted (stage 2). The second example is a symmetric pattern of multiple-seam mining with thicker interburden layers (Fig. 1d). The two upper panels in this model were extracted (stage 1) before commencement of the lower panel extraction (stage 2). Photographs, 3D scan of the model and surface subsidence measurements by optical transducers were captured after the end of each stage. To avoid repetition, the content already covered in the published work by Ghabraie et al. [16] is not reported here and the photogrammetry and DIC analysis results for case 2 only (Fig. 1d) are presented.

\subsection{Photogrammetry}

Photogrammetry is a popular, cheap and simple measurement method used in physical modelling of miningrelated problems and yet one of the most reliable measurement techniques if suitable equipment is used. There are various examples of this measurement method in physical modelling in the literature (Table 1). Only equipment required for this method is a high resolution camera. Using this method, photos at different stages of the test are compared with each other to derive the displacement vectors. The number of tracking points can be chosen in accordance with the purpose of the model and desired measurement precision (one point in every $5 \mathrm{~cm}$ in this study). These target points can then be used to extract displacement vectors between any two stages of the model. This is achieved by placing two photos of two stages of the model together and tracing the tracking points on the surface of the model (Fig. 2). Horizontal and vertical displacement at each point can then be derived by extrapolating the vertical and horizontal component of each displacement vector.

\subsubsection{Examples photogrammetry results}

In case of subsidence analysis, measurement of surface vertical (subsidence) and horizontal displacement profiles are of significant importance. Many of the key subsidence parameters can be measured based on the photogrammetry measurements of surface displacement profiles, such as, limit of the subsidence for each stage, maximum subsidence, subsidence factor, location of the maximum subsidence and incremental subsidence after each stage. Examples' photos used for photogrammetry analysis are shown in Fig. 3. Given the quality of these photos in this study, displacement vectors to an accuracy of approximately $-/+0.1 \mathrm{~mm}$ can be measured.

Figs. $3 c$ and $d$ show the profiles of surface vertical and horizontal displacement in the case 2 after stage 1 and 2. It can be seen that the magnitude of subsidence after the lower extraction is increased significantly, resulting in the maximum subsidence of approximately $62 \%$ of the combined extraction thickness of both layers above the overlapping parts of the panels. This observation agrees well with field observations of mining induced multipleseam subsidence [14]. Because of the presence of a thick interburden in this case the magnitude of surface measured horizontal displacement was relatively small (less than $0.1 \mathrm{~mm}$ ). These displacements are smaller than the minimum accuracy of the photogrammetry analysis $(-/+0.1 \mathrm{~mm})$. This is inferred to be the reason for observing fluctuations in the measured horizontal displacement in Fig. 3d. The accuracy of the results can definitely be improved by utilizing a higher resolution digital camera.

\subsection{Digital Image Correlation (DIC)}

DIC was first introduced during the 1980s by Sutton et al. [31,39]. It is a method to measure full-field deformation pattern of deformed samples [31, 32]. By having two photos of different stages of the test, 
displacement contours can be drawn by tracking number of target points in the reference photo and the deformed one [33]. A region of interest (ROI) is introduced in the beginning of the analysis and then the deformation is measured by optimizing the correlation between the target points in two (or more) images [32] (Fig. 4a). This analysis can be done using various algorithms. These algorithms have been discussed extensively by other researchers in the literature [39-41]. Based on these algorithms, a number of open source and free software packages and codes are available for researchers to be used for DIC analysis. In this paper NCORR_v1_1 was employed, which is a Matlab code developed by Justin Blaber.

DIC method's ability to produce colour coded displacement contour graphs is of great interest for researchers. This method of analysis provides researchers with a full-field view of the model's substrata movement pattern (both vertical and horizontal displacement). The visualized output of this method can be readily used to study the general mechanism of ground movement around an underground opening. In addition, DIC analysis only takes a few minutes to perform and can be done right after the test. Only basic knowledge of the DIC technique is required to perform the analysis. However, because of using limited number of target points to derive the final displacement contour, displacement measurements by DIC method is not as accurate as the photogrammetry.

\subsubsection{Examples DIC analysis results}

Results of the DIC substrata vertical deformation analysis of the two stages of the case 2 are shown in Figs. $4 \mathrm{~b}$ and $\mathrm{c}$. These results can be used to investigate the mechanism causing the strata layers deform after each stage of the test, locate maximum displacement, monitor the development of the ground movement from the coal seam to the surface, determine the limit of the subsidence and examine the effects of the lower extraction on the upper panel.

Figs. $4 \mathrm{~b}$ and c provide a clear illustration of the substrata vertical movement pattern after extraction of the upper and lower panels in case 2. It can be observed that the vertical strata deformation is increased above the upper panels after extraction of the lower panel in case 2 (Fig. 4c). In the area above the overlapping parts of the upper panels, because of the closure of existing cavities after the upper panels' extraction, substrata movement is almost doubled (compare Fig. 4b and c). Comparing the ground surface deformation pattern from DIC outputs with Fig. 3, indicates a reasonable correlation between DIC and photogrammetry results (compare surface deformation in Fig. 4c and Fig. 3c, for example).

\subsection{Optical Non-Contact Displacement Transducer (optoNCDT) devices}

optoNCDT devices are designed for one dimensional length measurements. A basic optical displacement transducer measures changes in length of an object to specific accuracy depending on the type of the device used. OptoNCDT using a laser beam light can measure the changes in distance from an object to fraction of a millimetre. Depending on the type of the optoNCDT, it can measure distance of an object between 0.5 to $2000 \mathrm{~mm}$ from the pointer [34]. The device projects a light beam to the measurement target, which is reflected back to the optical receiving position-sensitive element. If the position of the target point is changed, the location of the reflected light on the receiving element is changed accordingly. Figure 5 illustrates the working mechanism of an optoNCDT. The distance of the target point at any time can be measured using triangulation [42-43]. With this method, distance $D$ can be calculated as follow

$$
\frac{D}{E}=\frac{F}{G} \quad \text { Eq. } 3
$$


where $E$, is the distance from the pointer to the lens, $F$, is the distance from the lens to the receiving element, and $G$, is the measured distance from the original location are known (Fig. 5).

\subsubsection{Data acquisition and interpretation}

The data from optoNCDT devices can be easily transferred and recorded to a computer. With suitable software packages the data can be automatically recorded in user-defined time spans (or whenever the conductor manually starts recording the data). An important advantage of using optoNCDT compare to other measuring techniques is its ability to capture real time data during the time that the test is being conducted. This provides the researchers with the benefit of analysing the surface deformation data during the testing.

Monitoring the final static state of the model requires permitting the model to reach its final displacement state after performing each stage of the test. Similarity theory can be used to calculate the suitable time ratio between the real case and the laboratory test. This ratio then can be used to approximate the required time that should be left between each stage of the test. An easier, and yet more accurate method, is to monitor the dynamic surface subsidence until it reaches its final status after conducting each stage of the test. The optoNCDTs are capable of doing this by actively monitoring one dimensional displacement on number of fixed target points (Fig. 1a). Using this data, the final stage of the test can be determined when the displacement values converge to (stabilize at) a certain measurement (Fig. 6). Suitable data can then be captured at the final static stage of the test.

In addition to dynamic subsidence measurement, data from optoNCDT devices can be used as an alternative method to measure one dimensional deformation on any surface of the model. This method is quicker than other measurement methods and provides researchers with initial displacement measurements just after conducting the test.

\subsubsection{Examples dynamic vertical deformation measurements results}

Using optoNCDT devices, the dynamic vertical deformation of the model top surface during the test can be measured from the beginning of the test. According to the observations, after initiating the extraction, the strata take a few minutes to respond [35]. The caving process, then, starts in the area above the extraction and the deformation becomes evident on the ground surface (Fig. 6a). By extracting the whole panel, the ground movement is developed significantly on the ground surface. This is followed by a stabilization phase, the time in which the strata reach its final state and the ground movements stabilize.

This dynamic measurement of the surface deformation can indicate the final stage of the test (Fig. 6). This stage is considered as the appropriate time for the researchers to take the final data (photographs and 3D laser scan) for further analysis of the ground movement profile.

\subsection{Terrestrial Lase Scanner (TLS)}

TLS instruments can observe surface movement on numerous scales, including millimetres to tens of meters. These surfaces are generated from clouds of points or 'point clouds' consisting of a multitude of observed positions in the instruments coordinate system or other coordinate system (Fig. 7a). By repeatedly remeasuring these surfaces in the same coordinates system it is possible to measure displacement anywhere within the point cloud. This technology differs from optical transducers, which measure displacement, but only at discrete locations.

Modern TLS instruments generate point clouds by measuring the distance or range from the centre of the instrument to the target surface. This occurs by either measuring the time of flight (TOF) of the signal or the phase 
change of the reflected signal (Fig. 7b and c). This concept is based on Electromagnetic Distance Measurement and the range of the target can be found using the following equations using two methods of TOF (Eq. 4) and phase change (Eq. 5) [44]:

$$
\begin{array}{ll}
R=v \times t / 2 & \text { Eq. } 4 \\
R=(M \lambda+\Delta \lambda) / 2 & \text { Eq. } 5
\end{array}
$$

where $R$ is the distance or range, $v$ is the speed of electromagnetic radiation (known value), $t$ is the measured travel time, $M$ is the integer number of wavelengths, $\lambda$ is the value of the wavelength (known value) and $\Delta \lambda$ is the fractional part of the wavelength $(\varphi / 2 \pi)$, where $\varphi$ is the phase angle. Each measurement type is more suited to specific applications. The TOF approach can measure over very large distances (e.g. $300 \mathrm{~m}$ ), but is slower and less accurate than phase based observations. Phase based observations are rapid and accurate but presently limited to shorter ranges. Newer instruments, such as hybrid phase-pulse laser scanners combine the range and low noise sensitivity of the time of flight technique, with the high accuracy ( $\sim 2 \mathrm{~mm}$ at $30 \mathrm{~m})$ at short range of phase shift technology [36]. Unlike photographs, which are made of coloured points (pixels), the point cloud is a cluster of measurement points. Each point is defined by an $X-Y-Z$ coordinates information. This makes it possible to view the point cloud from different angles by simply changing the local coordinate system (manipulating the point cloud scene).

TLS and Airborne Terrestrial Laser Scanning (ALS) have already been employed for mine subsidence measurements in Australia. Examples include subsidence mapping at Illawarra coal mine [37] and near the city of Wollongong [38]. However, there are very few examples of the application of TLS for laboratory-based scaled physical subsidence modelling and only recently has TLS been used for this purpose [16, 22].

\subsubsection{Surface subsidence and cavity growth mapping by TLS}

Modern TLS are capable of measurements at rates of hundreds of thousands of observations a second, generating massive 3D point cloud scenes. Using these devices, conducting a high resolution 3D scan of the whole physical model takes only a few minutes. This makes it possible to conduct a 3D scan after completing of each stage of the physical modelling. Various 3D scans of different stages of the test can then be compared and desired parameters can be extracted. Also, the ability to view the model (point cloud) from various angles provides engineers with the possibility to investigate the results in a 3D environment when the model is no longer available [16].

One dimensional surface deformation can be easily measured on a section of the point cloud, in a timely manner, by comparing the point clouds after each stage of the test. This can be done by using point cloud software, such as Trimble Realworks (), which puts together the two chosen surfaces and map the surface deformation (Fig. $8 a)$.

In addition to the common practice of laser scanning, a new technique has been developed and is introduced in this paper to map the cavity growth pattern on any surface of the model. This technique is based on the working mechanism of the TLS, that the signal sent from the laser scanner can reach the depth of the cracks in the model, if the laser scanner is located at an appropriate location. The wider the fracture's width is, the greater its depth is and, thus, the farther the signal can reach (with the maximum of model's thickness). Therefore, considering the surface of the model to be on $X-Y$ plane (in local coordinates of the laser scanner), $Z$ axis indicates the depth of 
the cavities. By assigning colours to different $Z$ values, projection of each cavity on $X-Y$ plane can be drawn (Fig. 8b). Warm and cold colours can be assigned to deep and shallow cavities respectively. In Fig. 8b, for instance, negative values on $Z$ axis indicate deep cavities, i.e. warm colour (red), and $z=0$ indicates zero cavity (any point on X-Y plane), i.e. cold colour (blue).

The cavity growth maps for different stages of the test can be compared together to monitor the fracture development pattern. An important factor in achieving high quality cavity growth maps is the resolution of the scan. If the resolution is equal or less than the fractures aperture in the model, some of the fractures would not appear in the point cloud. For example, if the laser scanner has the resolution of $2 \mathrm{~mm}$, fractures with aperture of $2 \mathrm{~mm}$ or less cannot be modelled appropriately.

\subsubsection{Examples cavity growth mapping results}

The introduced cavity growth mapping technique has been used for the first time in physical modelling of a mining-related problem. The colour coding of the cavity growth map makes it possible to investigate the changes in fractures size and aperture by comparing two stages of the model. Figs. 9a and b indicate that the depth and aperture of the fractures above the upper panels in both examples are severely different in stage one and two.

The crack propagation pattern and the influence of one extraction on the existing cracks around another extraction are of great importance in mining engineering. It can be seen that in both examples, fractures in overburden in the area above and close to the overlapping parts of the panels become closed after the stage two for both models (Figs. 9a and b). It is also visible that areas above the outer parts of the upper panels in both models are less altered than the area above the overlapping panels.

\subsection{Hybrid TLS-DIC technique}

Producing the colour coded displacement contours of a surface of the model from the point cloud, similar to what the DIC method can produce, is prone to error, extremely time consuming and in many cases impossible to perform. On the other hand, the ground surface vertical deformation contour produced by TLS has significantly better accuracy than the DIC output. However, by combining these two methods, a highly accurate ground surface vertical deformation and a full-field substrata movement profile can be achieved. By using image editing programs, the ground surface deformation contour by TLS and the DIC substrata movement profile can be combined to produce a 3D view of the whole model's subsidence profile (Fig. 10a). This hybrid approach is easy to perform in a timely manner and at the same time provides engineers with an accurate 3D illustration of surface subsidence profile and substrata movement pattern. Also, the combined view of the displacement contour of both examples show that the laser scanner ground surface deformation and DIC measurements agree well.

\subsection{Comparison of different measurement techniques}

One of the advantages of using various measurement techniques is that they can be used for cross-checking and elimination of possible errors that may occur during the data collection. Fig. 10b illustrate that all the three measurement methods correlate resonably well in measuring the surface vertical deformation profile. The photogrammetry and laser scanner output are commonly more accurate than the optical transducers. This is because of the set-up of the model test and that the optical transducers are not fixed in one location and they need to be relocated on the sliding bar to monitor number of target points (Fig. 1). This make them vulnerable to vibrations and thus less accurate measurement. 


\section{Physical modelling protocol}

The proposed combination of measuring devices and the set-up of the physical test explained in the previous sections provide researchers with a variety of output parameters. Each measurement method monitors specific ground subsidence parameters used by subsidence engineers. The DIC method can produce full-field displacement map of the model while photogrammetry can extract accurate displacement measurement for all the target points on the model surface. In addition, dynamic subsidence measurement by optoNCDT becomes extremely useful at the time of performing the physical modelling and can produce real time displacement readings on desired points. It can as well be utilized to determine an appropriate time for data taking at the end of each stage of the test. The new method of cavity growth mapping can precisely illustrate the fracture growth pattern around an underground excavation. With high-tech laser scanning devices, performing a close range 3D scan of the model test with the resolution of a fraction of a millimetre is possible in a timely manner. This provides engineers with the ability to monitor the finest cracks with width of less than $1 \mathrm{~mm}$ on the model surface at different stages of the test and produce a detailed map of the fracture propagation around an underground excavation.

The combination of these measurement devices, together with other available monitoring techniques, can also be used in studying various mining-related problems. Various data acquisition devices and methods of analysis can be utilized to acquire a range of output parameters. The decision to choose appropriate monitoring devices and method of analysis rely heavily on the purpose of the study. A physical model can be constructed based on the similarity theory rules with suitable material properties, size and boundary conditions. Then appropriate data acquisition devices and analysing techniques can be selected in accordance with the role they play in meeting the aim of the study. Under this process only, physical modelling technique would serve to tackle an issue in an efficient manner. Fig. 11 shows a physical modelling protocol for mining-related problems based on the previously published works (Table 1) and introduced methods in previous sections.

In addition to the purpose of a study, choosing suitable measuring device depends on other factors, such as, the quality of the output, accuracy and processing time. These factors can be significantly different between each method. For example, to monitor the cavity growth and failure/cracking process, 3D laser scanner, Acoustic Emission (AE) measuring system and infrared thermography technique can be used. The signal from AE measurement system can depict the transition from elastic to plastic. It can also show the occurrence of local cracking around discrete $A E$ sensors at different times [23]. But, AE measurements are unable to produce an illustrative output. Infrared thermography requires complicated calculation process [21]. The cavity growth mapping with 3D laser scanner can precisely monitor full-field cracking and caving process in a timely manner, however it cannot show the failure areas as clear as the AE measuring system.

Another factor that should be considered in designing an experimental set-up is that some of the methods are able to produce secondary outputs. For instance, photogrammetry can be used to derive full-field displacement contours with extra effort if sufficient target points are placed on the target surface of the model. Photogrammetry can also be used for monitoring cracking by visual inspection of the photographs at different stages of the modelling. However, achieving secondary outputs are deemed to increase the computational cost and effort.

By means of the physical modelling protocol shown in Fig. 11 one could determine suitable measures, which can produce the desired parameters, and address the purpose of a study. For example, to study the caving process 
around an underground working panel, researchers would need to measure stress, monitor failure and crack propagation patterns in the surrounding rock (see the connections between red and blue parts in Fig. 11). To achieve this, stress/strain gauges could be used to measure stress along with the laser scanner, AE measuring system or infrared thermography for failure and crack propagation monitoring (see the connections between blue and green parts in Fig. 11). Accuracy, analysing time and discrete/full-field measurement output of these devices also need to be considered in order to obtain the most suitable output.

\section{Further application of the physical modelling protocol}

The proposed physical testing protocol is believed to have broad application in physical modelling of miningrelated problems. The monitoring system of combination of photography, optical transducers and TLS, together with dynamic subsidence measurement, photogrammetry, DIC and cavity growth analysing methods has the ability to monitor the surface and subsurface displacement profile for a vast area related to underground mining and tunnelling. By utilizing number of modifications to the proposed physical modelling set-up, many other miningrelated issues can be physically modelled. For instance, optoNCDT devices can be used in monitoring the convergence or displacement on inner borders of an underground excavation by installing them on a standing pillar and pointing the light beam on the surface of the excavation (Fig. 12). With this set-up, real time dynamic deformation of the excavation's surface during the testing can be measured. The in-situ stress condition can be modelled by applying surface load on each side of the frame. If surface subsidence needs to be investigated, optoNCDT devices can be installed on a sliding bar and dynamic surface subsidence measurements can be captured. Target points should be placed on the front and top surface of the model for proper photogrammetry analysis. 3D TLS and a digital camera must be placed in front of the model and close to the centre of the model to reduce the image distortion. The proposed physical modelling protocol has no restriction in using strain/stress gauges. These can be readily installed within the material without interfering with other measurement devices.

The proposed protocol is particularly useful for surface and subsurface deformation analysis after any type of underground excavation or mining activity. Some examples of various applications of the proposed testing protocol include:

- Shallow tunnelling displacement and associated surface displacement;

- Tunnel face/roof failure and associated surface displacement;

- Single and multiple-seam mining substrata deformation, crack propagation and subsidence analysis;

- Cavern stability analysis.

\section{Conclusions}

Application of some recent optical and laser based measurement devices together with number of data analysis methods for physical modelling of mining-induced subsidence have been presented in this paper. Two examples of physical modelling of multiple-seam mining-induced ground subsidence are modelled and appropriate parameters are recorded using a digital camera, an Optical Non-Contact Displacement Transducer (optoNCDT) and a 3D Terrestrial Lase Scanner (TLS). With the proposed physical test set-up and data interpretation methods, authors were able to perform and obtain:

- Photogrammetry analysis - discrete vertical and horizontal displacement measurement at various points

- Digital Image Correlation (DIC) analysis - a full-field substrata displacement measurement 
- Dynamic surface subsidence measurement by use of optical transducers

- Surface subsidence measurement by 3D TLS, photogrammetry and optical transducers and

- Cavity growth mapping by means of 3D scan of the model.

In addition, a physical modelling protocol has been introduced that takes into account number of commonly used monitoring techniques together with newly developed methods. This includes an innovative approach of cavity growth mapping, which has been used for the first time in modelling a mining related problem, and dynamic surface deformation monitoring.

By using the proposed protocol, researchers would be able to choose an appropriate combination of monitoring devices in accordance with the purpose of a study. The precision of each method, analysing time and the ability to produce full-field/discrete readings are also considered in the proposed protocol. It is hoped that this protocol would assist researchers in the field of underground mining and tunnelling with a practical and easy way to configure an appropriate experimental set-up and achieve the desired output.

\section{Acknowledgement}

The authors wish to acknowledge Xiangyang Zhang, who helped performing the physical experiments reported in this paper.

\section{References}

[1] Li Z, Liu H, Dai R, Su X. Application of numerical analysis principles and key technology for high fidelity simulation to 3-D physical model tests for underground caverns. Tunnelling and Underground Space Technology. 2005;20(4):390-399.

[2] Ashby MF. Physical modelling of materials problems. Materials Science and Technology. 1992;8:102-111.

[3] Whittaker BN, Gaskell P, Reddish DJ. Subsurface ground strain and fracture development associated with longwall mining. Mining Science and Technology. 1990;10(1):71-80.

[4] Sun T, Yue Z, Gao B, Li Q, Zhang Y. Model test study on the dynamic response of the portal section of two parallel tunnels in a seismically active area. Tunnelling and Underground Space Technology.2011; 26(2):391397.

[5] Weishen Z, Yong L, Shucai L, Shugang W, Qianbing Z. Quasi-three-dimensional physical model tests on a cavern complex under high in-situ stresses. Int. J. Rock Mech. Min. Sci. 2011;48(2):199-209.

[6] Ren W, Guo C, Peng Z, Wang Y. Model experimental research on deformation and subsidence characteristics of ground and wall rock due to mining under thick overlying terrane. Int. J. Rock Mech. Min. Sci. 2010;47(4):614-624.

[7] Lee Y-J, Bassett RH. Influence zones for 2D pile-soil-tunnelling interaction based on model test and numerical analysis. Tunnelling and Underground Space Technology. 2007;22(3):325-342.

[8] Whittaker BN, Reddish DJ. SubsidenceOccurrence, Prediction and Control. Developments in Geotechnical Engineering, ed. N.W. Barry and J.R. David. Vol. 56. Elsevier; 1989.

[9] Sutherland HJ, Heckes AA, Taylor LM. Physical and numerical simulations of subsidence above high extraction coal mines. In: ISRM Symposium, editors: Brown ET and Hudson J.A. Cambridge, UK; 1984. P. 6572. 
[10] Wold MB. Blocky physical model of longwall caving under strong roof conditions. In: $26^{\text {th }}$ US Symposium on Rock Mechanics. Rapid City, SD; 26-28 Jun 1985. p. 1007-1014.

[11] Suchowerska AM, Carter J.P, Merifield RS. Horizontal stress under supercritical longwall panels. Int. J. Rock Mech. Min. Sci.2014;70(0):240-251.

[12] Sui W, Hang Y, Ma L, Wu Z, Zhou Y, Long G, et al. Interactions of overburden failure zones due to multipleseam mining using longwall caving. Bulletin of Engineering Geology and the Environment. 2014;1-17.

[13] Luo Y, Qiu B. CISPM-MS: A Tool to Predict Surface Subsidence and to Study Interactions Associated with Multi-Seam Mining Operations. In: 31st International Conference on Ground Control in Mining. Morgantown, WV. 2012. p. 1-7.

[14] Li G, Steuart P, Pâquet R, Ramage R. A Case Study on Mine Subsidence Due to Multi-Seam Longwall Extraction. In: Second Australasian Ground Control in Mining Conference. Sydney, NSW. 2010.

[15] Unlu T, Akcin H, Yilmaz O. An integrated approach for the prediction of subsidence for coal mining basins. Engineering Geology. 2013;166:186-203.

[16] Ghabraie B, Ren G, Zhang X, Smith J. Physical modelling of subsidence from sequential extraction of partially overlapping longwall panels and study of substrata movement characteristics. International Journal of Coal Geology. 2015;140(0):71-83.

[17] Suchowerska AM, Merifield RS, Carter JP. Vertical stress changes in multi-seam mining under supercritical longwall panels. Int. J. Rock Mech. Min. Sci. 2013;61(0):306-320.

[18] Zhang M, Shimada H, Sasaoka T, Matsui K, Dou L. Evolution and effect of the stress concentration and rock failure in the deep multi-seam coal mining. Environmental Earth Sciences. 2014;72(3):629-643.

[19] Whittaker BN, Reddish DJ, Fitzpatrick DJ. Ground fractures due to longwall mining subsidence. In: International Mine Water Association Preceedings. Spain 1985. p. 1057-1072.

[20] Park S-H, Adachi T. Laboratory model tests and FE analyses on tunneling in the unconsolidated ground with inclined layers. Tunnelling and Underground Space Technology. 2002;17(2):181-193.

[21] He M, Jia X, Gong W, Faramarzi L. Physical modeling of an underground roadway excavation in vertically stratified rock using infrared thermography. Int. J. Rock Mech. Min. Sci. 2010;47(7):1212-1221.

[22] Thongprapha T, Fuenkajorn K, Daemen JJK. Study of surface subsidence above an underground opening using a trap door apparatus. Tunnelling and Underground Space Technology. 2015;46(0):94-103.

[23] Lin P, Liu H, Zhou W. Experimental study on failure behaviour of deep tunnels under high in-situ stresses. Tunnelling and Underground Space Technology. 2015;46(0):28-45.

[24] Piede L. Model test study on double lining of tunnels. Tunnelling and Underground Space Technology. 1986;1(1):53-58.

[25] Xie GX, Chang JC, Yang K. Investigations into stress shell characteristics of surrounding rock in fully mechanized top-coal caving face. Int. J. Rock Mech. Min. Sci. 2009;46(1):172-181.

[26] Huayang D, Xugang L, Jiyan L, Yixin L, Yameng Z, Weinan D, et al. Model study of deformation induced by fully mechanized caving below a thick loess layer. Int. J. Rock Mech. Min. Sci. 2010;47(6):1027-1033. 
[27] Liu Y-k, Zhou F-b, Liu L, Liu C, Hu S-y. An experimental and numerical investigation on the deformation of overlying coal seams above double-seam extraction for controlling coal mine methane emissions. International Journal of Coal Geology. 2011;87(2):139-149.

[28] Huang F, Zhu H, Xu Q, Cai Y, Zhuang X. The effect of weak interlayer on the failure pattern of rock mass around tunnel - Scaled model tests and numerical analysis. Tunnelling and Underground Space Technology. 2013;35(0):207-218.

[29] Lei M, Peng L, Shi C. Model test to investigate the failure mechanisms and lining stress characteristics of shallow buried tunnels under unsymmetrical loading. Tunnelling and Underground Space Technology. 2015;46(0):64-75.

[30] Li SC, Wang Q, Wang H.T, Jiang B, Wang D.C, Zhang B, et al. Model test study on surrounding rock deformation and failure mechanisms of deep roadways with thick top coal. Tunnelling and Underground Space Technology. 2015;47(0):52-63.

[31] Kashfuddoja M, Prasath RGR, Ramji M. Study on experimental characterization of carbon fiber reinforced polymer panel using digital image correlation: A sensitivity analysis. Optics and Lasers in Engineering. 2014;62(0):17-30.

[32] Zhou Y, Sun C, Song Y, Chen J. Image pre-filtering for measurement error reduction in digital image correlation. Optics and Lasers in Engineering. 2015;65(0):46-56.

[33] Jiang L, Xie H, Pan B. Speeding up digital image correlation computation using the integral image technique. Optics and Lasers in Engineering. 2015;65(0):117-122.

[34] Micro-Epsilon user manual, Application Giud - Laser Triangulation Sensors optoNCDT.

[35] Kratzsch H. Mining Subsidence Engineering. Berlin, Heidelberg: Springer-Verlag; 1983.

[36] Trimble Navigation, L. CX Laser Scanner Datasheet. 2015 [cited 2015; Available from: http://www.trimble.com/3d-laser-scanning/cx.aspx?dtID=support].

[37] Riley P, Crowe P. Airborne and Terrestrial Laser Scanning - Applications for Illawarra Coal. In: Aziz, N (ed), Coal Operators' Conference2006. University of Wollongong \& the Australasian Institute of Mining and Metallurgy, Wollongong, NSW, 2006. p. 266-275.

[38] Palamara DR, Nicholson M, Flentje P, Baafi E, Brassington GM. An evaluation of airborne laser scan data for coalmine subsidence mapping. International Journal of Remote Sensing. 2007;28(15):3181-3203.

[39] Sutton MA, Wolters WJ, Peters WH, Ranson WF, McNeill SR. Determination of displacements using an improved digital correlation method. Image and Vision Computing, 1983;1(3):133-139.

[40] Sutton MA, Orteu JJ, Schreier HW. Image correlation for shape and deformation measurements, basic concepts, theory and applications. New York, NY,US: Springer; 2009.

[41] Rastogi KP. Photomechanics, topics in applied physics. Berlin, Germany: Springer; 2000 (ISBN 3-540-65990$0)$.

[42] Girao PMBS, Postolache OA, Faria JAB, Pereira JMCD. An overview and a contribution to the optical measurement of linear displacement. Sensors Journal, IEEE, 2001;1(4):322-331.

[43] Berkovic G, Shafir E. Optical methods for distance and displacement measurements. Advances in Optics and Photonics, 2012;4(4):441-471. 
Taylor \& Francis Group; 2009.

\section{List of Figures and Tables:}

Table 1 Summary of model materials and measurement systems.

Table 2 Model parameters and similarity constants.

Table 3 Technical specification and measurement precision of monitoring devices.

Fig. 1. a) Boundary conditions and front view of the model test frame, b) top view of the model test and set-up of the TLS, camera and optoNCDT, c) Multiple-seam mining configuration of Example 1 and d) Example 2.

Fig. 2. Principles of photogrammetry analysis.

Fig. 3. Photos of Example 2 after completing a) stage 1 and b) stage 2 used for photogrammetry analysis, c) Example 2 subsidence and d) horizontal displacement of the ground surface by photogrammetry.

Fig. 4. a) Principles of DIC analysis, b) DIC output of Example 2 substrata vertical displacement contours after stage 1 and c) stage 2 .

Fig. 5. Working mechanism of an optoNCDT (adapted from [43]).

Fig. 6. Incremental dynamic surface subsidence of stage 2 at different surface locations for a) Example 1 and b) Example 2, where $X$ is the target point's horizontal distance from the middle of the model.

Fig. 7. a) An example point cloud from 3D TLS (Example 2 in Section 5.1), b) Laser scanner measurement of $R$ by means of TOF and c) phase change (adapted from [44]).

Fig. 8. a) One dimensional displacement measurement on a model's top surface and b) cavity growth mapping from the point cloud.

Fig. 9. Cavity growth mapping of stage 1 and 2 of a) Example 1 and b) Example 2.

Fig. 10. a) Hybrid TLS-DIC technique output for surface and substrata vertical deformation contours of stage 2 of Example 2 and b) Comparison of photogrammetry, optoNCDT and laser scanner's surface subsidence measurement for Example 2.

Fig. 11. Physical modelling protocol.

Fig. 12. An example modification of the proposed physical modelling protocol for studying strata and surface movement characteristics around an underground excavation. 


\begin{tabular}{|c|c|c|c|c|}
\hline $\begin{array}{l}\text { Measurement devices and } \\
\text { techniques }\end{array}$ & Acquired parameter & Purpose of model & Model material & Authors \\
\hline Photogrammetry & $\begin{array}{l}\text { Subsidence curve, } \\
\text { fracture propagation, } \\
\text { principal strain }\end{array}$ & $\begin{array}{l}\text { Subsidence } \\
\text { analysis }\end{array}$ & Sand-plaster-water & $\begin{array}{l}\text { Whittaker et al. }[3,19] \\
\text { and Whittaker and } \\
\text { Reddish }[8]\end{array}$ \\
\hline Photogrammetry & $\begin{array}{l}\text { Displacement, caving } \\
\text { process }\end{array}$ & $\begin{array}{l}\text { Multiple-seam } \\
\text { stability }\end{array}$ & Sand-plaster-water & Wold [10] \\
\hline $\begin{array}{l}\text { Photogrammetry - strain } \\
\text { gauges - flexural gauges }\end{array}$ & $\begin{array}{l}\text { Displacement, stress, } \\
\text { loads on lining }\end{array}$ & Tunnel stability & $\begin{array}{l}\text { Sand, talcum, } \\
\text { gypsum, etc. }\end{array}$ & Piede [24] \\
\hline $\begin{array}{l}\text { Load cells - laser displacement } \\
\text { sensor }\end{array}$ & $\begin{array}{l}\text { Earth pressure, } \\
\text { surface settlement }\end{array}$ & $\begin{array}{l}\text { Distribution and } \\
\text { variation of the } \\
\text { earth pressure } \\
\text { and surface } \\
\text { settlement after } \\
\text { tunnelling }\end{array}$ & $\begin{array}{l}\text { Aluminium rods } \\
\text { and blocks }\end{array}$ & Park and Adachi [20] \\
\hline $\begin{array}{l}\text { Mini-multi-point } \\
\text { extensometer - strain gauge - } \\
\text { optical fiber sensor meter - } \\
\text { acoustic measurement system }\end{array}$ & $\begin{array}{l}\text { Displacement, yield } \\
\text { zones, cavern inner } \\
\text { destruction }\end{array}$ & $\begin{array}{l}\text { Stability of an } \\
\text { underground } \\
\text { cavern (3D) }\end{array}$ & $\begin{array}{l}\text { Material similar to } \\
\text { the original rock }\end{array}$ & Li et al. [1] \\
\hline $\begin{array}{l}\text { Photogrammetry (VMS and } \\
\text { EngVis software) }\end{array}$ & Displacement & $\begin{array}{l}\text { Pile-soil-tunnelling } \\
\text { interaction }\end{array}$ & $\begin{array}{l}\text { Multi-sized } \\
\text { aluminium rods }\end{array}$ & Lee and Bassett [7] \\
\hline $\begin{array}{l}\text { Photogrammetry - strain } \\
\text { gauges }\end{array}$ & Stress distribution & Top coal caving & $\begin{array}{l}\text { Sand, gypsum, lime } \\
\text { and loess }\end{array}$ & Xie et al. [25] \\
\hline Photogrammetry - dial gauges & Displacement & $\begin{array}{l}\text { Rock deformation } \\
\text { around block } \\
\text { caving extraction }\end{array}$ & $\begin{array}{l}\text { Barite powder, iron } \\
\text { powder, glycerol, } \\
\text { etc. }\end{array}$ & Ren et al. [6] \\
\hline $\begin{array}{l}\text { Infrared thermography - } \\
\text { strain measurement }\end{array}$ & $\begin{array}{l}\text { Fractured and failure } \\
\text { zones }\end{array}$ & Roadway tunnel & Gypsum, water & He et al. [21] \\
\hline Photogrammetry & Displacement & $\begin{array}{l}\text { Subsidence } \\
\text { analysis }\end{array}$ & $\begin{array}{l}\text { Aggregates, lime } \\
\text { and gypsum }\end{array}$ & Huayang et al. [26] \\
\hline $\begin{array}{l}\text { Photogrammetry - } \\
\text { displacement sensors }\end{array}$ & Displacement & $\begin{array}{l}\text { Gas in multiple- } \\
\text { seam }\end{array}$ & $\begin{array}{l}\text { Plaster, grits, soot, } \\
\text { etc. }\end{array}$ & Liu et al. [27] \\
\hline $\begin{array}{l}\text { Photogrammetry - stress } \\
\text { sensors }\end{array}$ & $\begin{array}{l}\text { Displacement, ground } \\
\text { stress, failure shape }\end{array}$ & Tunnel stability & $\begin{array}{l}\text { Barite, sand, } \\
\text { plaster, etc. }\end{array}$ & Huang et al. [28] \\
\hline Photogrammetry -LVDT & $\begin{array}{l}\text { Displacement, caving } \\
\text { process }\end{array}$ & $\begin{array}{l}\text { Longwall caving } \\
\text { and water flow }\end{array}$ & $\begin{array}{l}\text { Sand, barite, } \\
\text { gypsum, etc. }\end{array}$ & Sui et al. [12] \\
\hline Laser scanner & Surface settlement & $\begin{array}{l}\text { Subsidence } \\
\text { analysis (3D) }\end{array}$ & Gravel & Thongprapha et al. [22] \\
\hline $\begin{array}{l}\text { Photogrammetry - wire strain } \\
\text { gauges - steel wire pressure } \\
\text { cells }\end{array}$ & $\begin{array}{l}\text { Fracture } \\
\text { development, } \\
\text { structural stress, rock } \\
\text { pressure }\end{array}$ & Tunnel stability & Clay, slag and sand & Lei et al. [29] \\
\hline $\begin{array}{l}\text { Photogrammetry - FBG - } \\
\text { multi-point extensometer }\end{array}$ & $\begin{array}{l}\text { Displacement, stress, } \\
\text { rock failure }\end{array}$ & $\begin{array}{l}\text { Roadway tunnel } \\
\text { stability }\end{array}$ & $\begin{array}{l}\text { Siltstone, silica } \\
\text { aggregates, gypsum, } \\
\text { etc. }\end{array}$ & Li et al. [30] \\
\hline $\begin{array}{l}\text { Photogrammetry - strain } \\
\text { gauges - displacement meters } \\
\text { - Acoustic emission (AE) }\end{array}$ & $\begin{array}{l}\text { Displacement, strain, } \\
\text { cracking behaviour }\end{array}$ & Tunnel stability & $\begin{array}{l}\text { Barite powder, } \\
\text { sand, expansion soil }\end{array}$ & Lin et al. [23] \\
\hline
\end{tabular}


Table 2

\begin{tabular}{lccc}
\hline & $\begin{array}{l}\text { Density } \\
(\mathrm{kg} / \mathrm{m} 3)\end{array}$ & $\begin{array}{l}\text { Strength } \\
(\mathrm{kPa})\end{array}$ & $\begin{array}{l}\text { Geometry } \\
(\mathrm{m})\end{array}$ \\
\hline Real case & 2700 & 16700 & 150 \\
Model test & 1909 & 52 & 0.66 \\
Similarity constants & 1.41 & 319.5 & $1 / 226$ \\
\hline
\end{tabular}


Table 3

\begin{tabular}{|c|c|c|c|}
\hline Device & $\begin{array}{l}\text { Distance to } \\
\text { model }\end{array}$ & $\begin{array}{l}\text { Measurement } \\
\text { precision }\end{array}$ & $\begin{array}{l}\text { Output } \\
\text { type }\end{array}$ \\
\hline TLS & $\sim 2 \mathrm{~m}$ & $\sim 2 \mathrm{~mm}$ at $\leq 30 \mathrm{~m}$ & digital-3D \\
\hline Camera & $\sim 2 \mathrm{~m}$ & 10.2 MP & digital-2D \\
\hline optoNCDT & $\sim 0.02-0.2 \mathrm{~m}$ & $0.001 \mathrm{~mm}$ & digital-1D \\
\hline
\end{tabular}




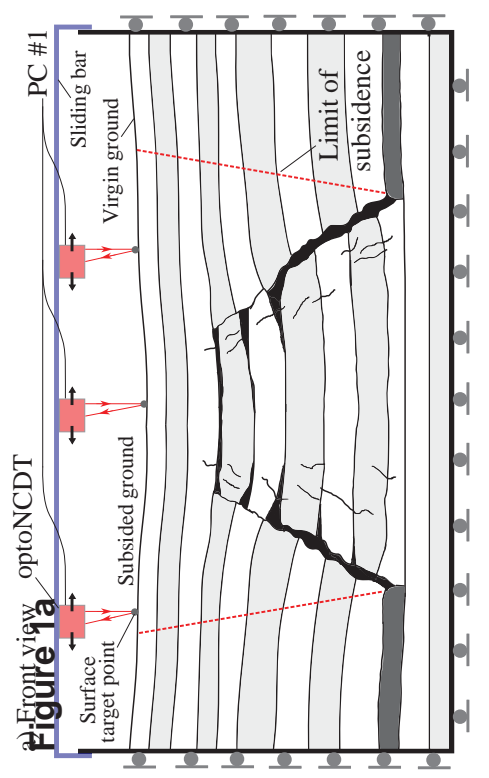




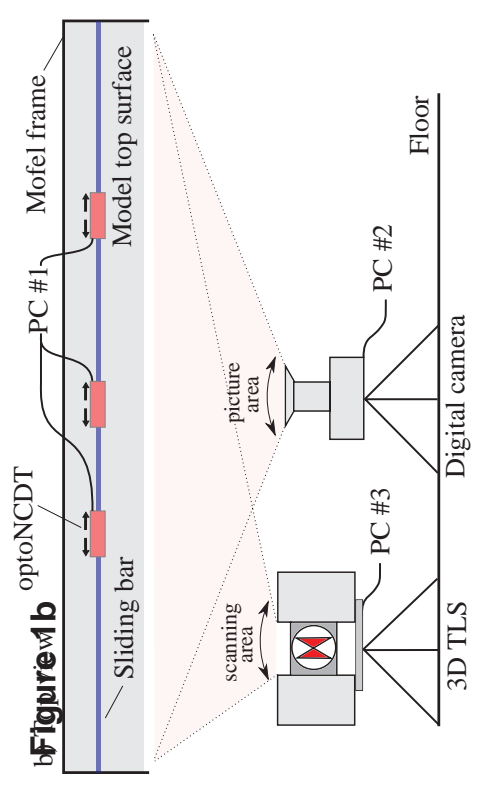




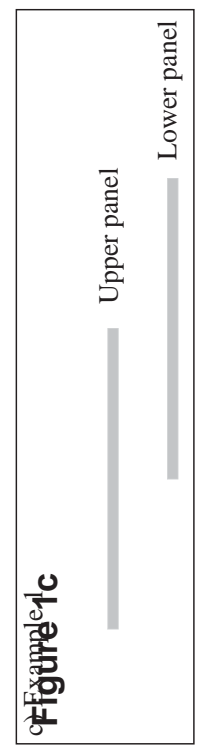




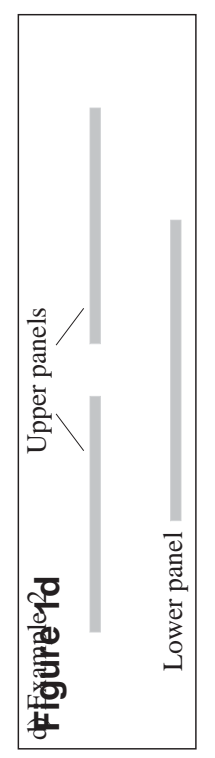




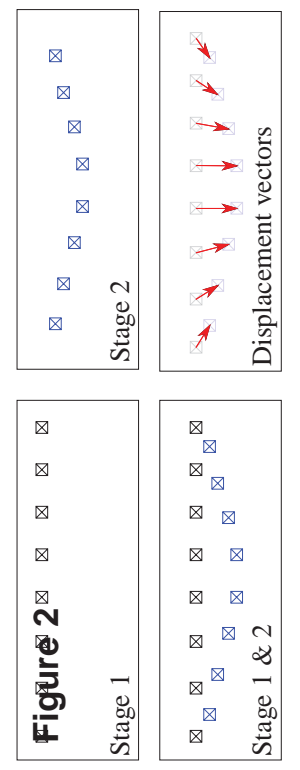




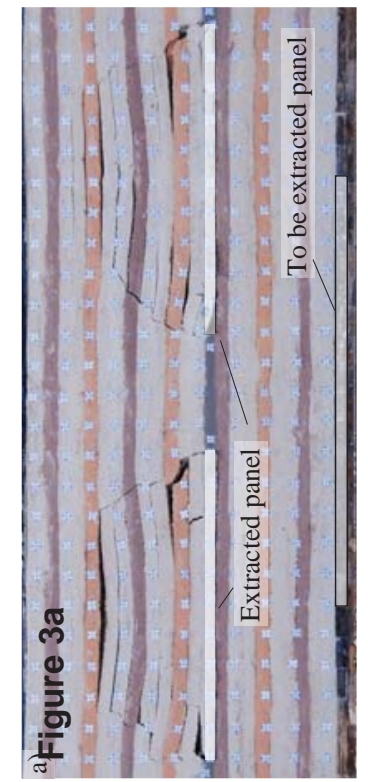




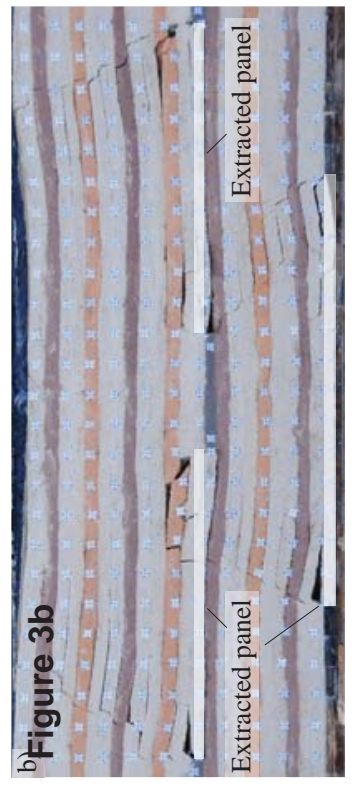




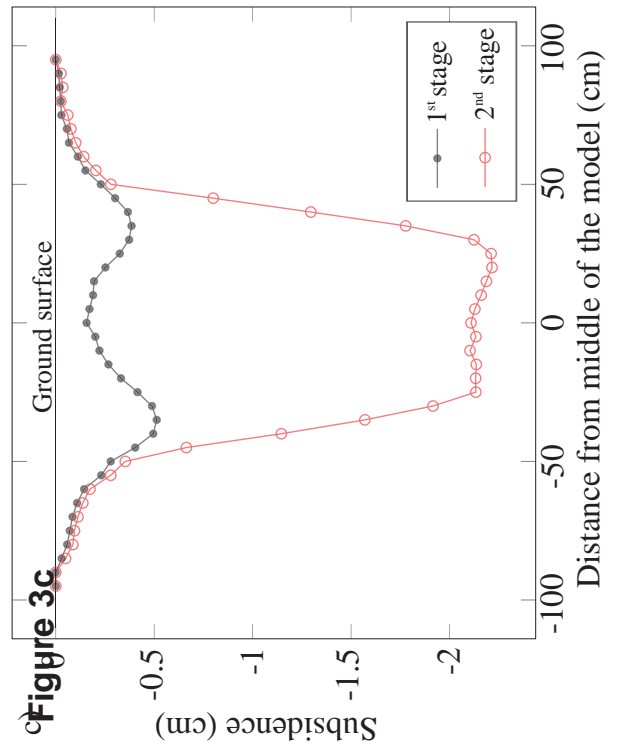




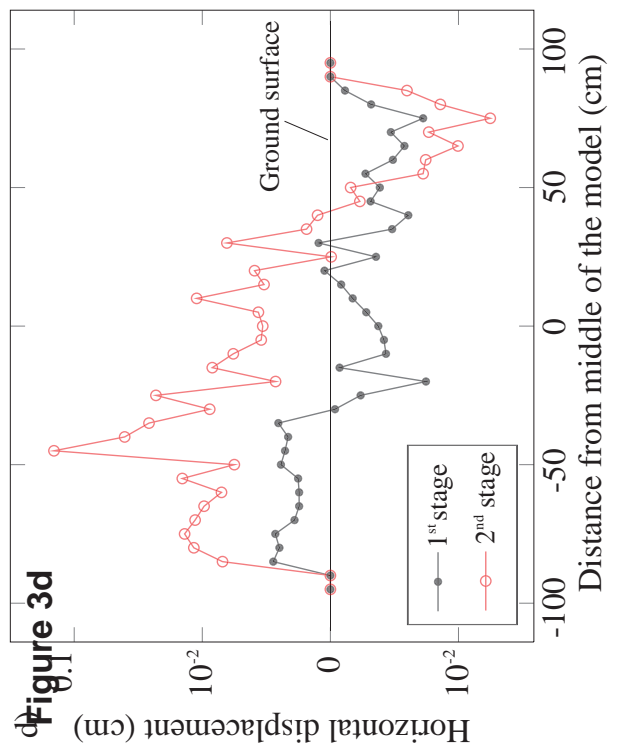




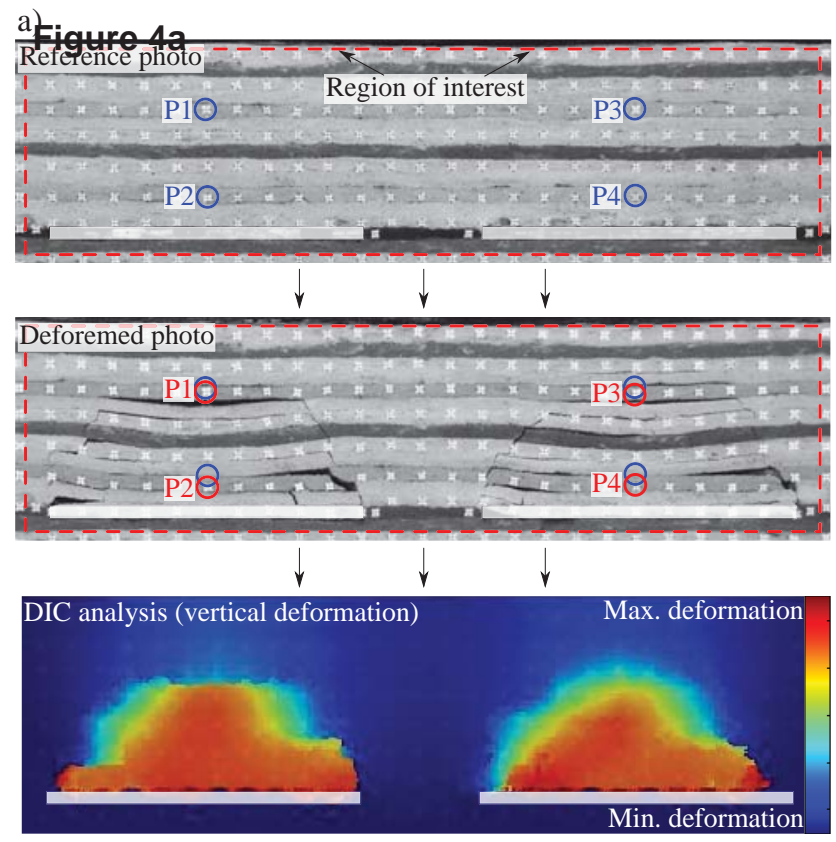




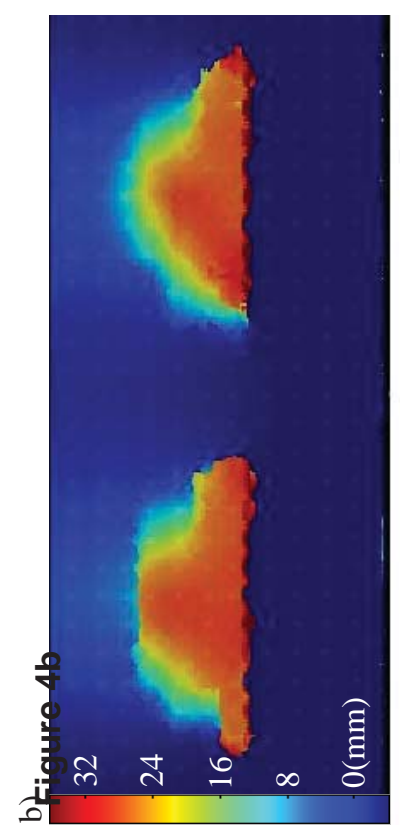




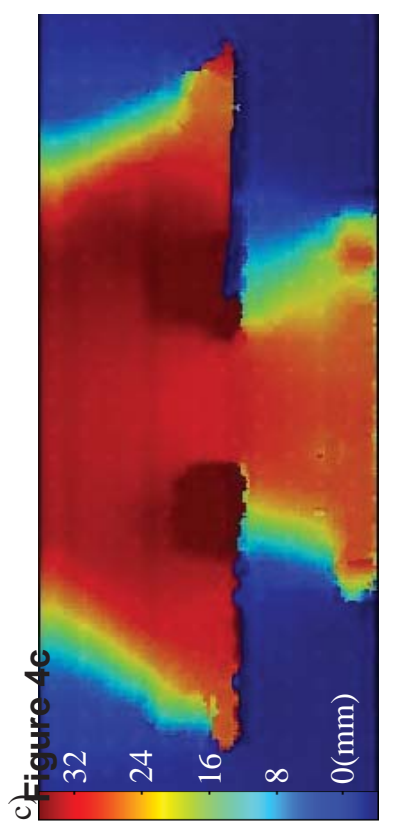




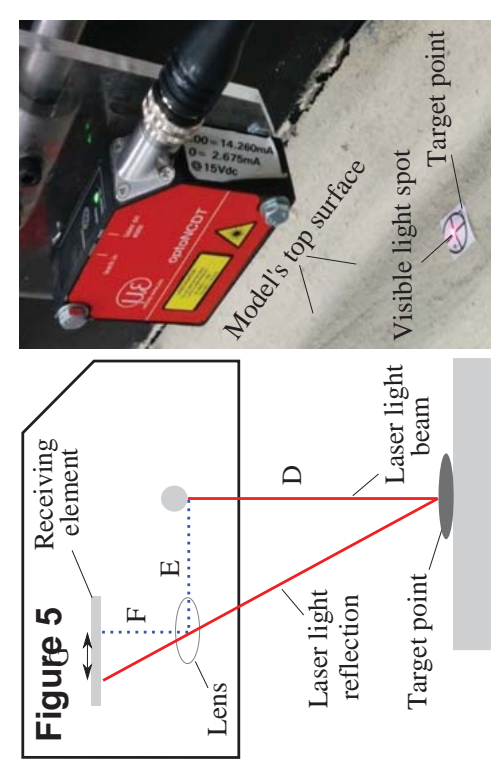




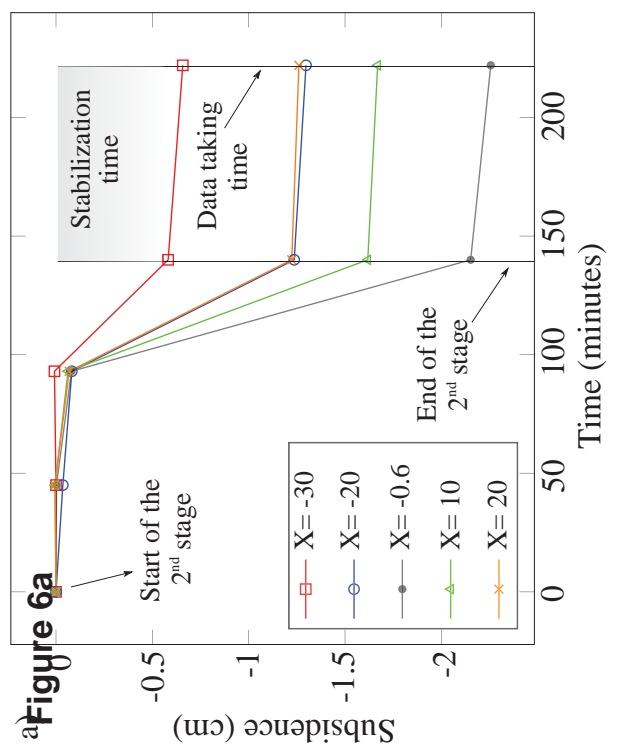




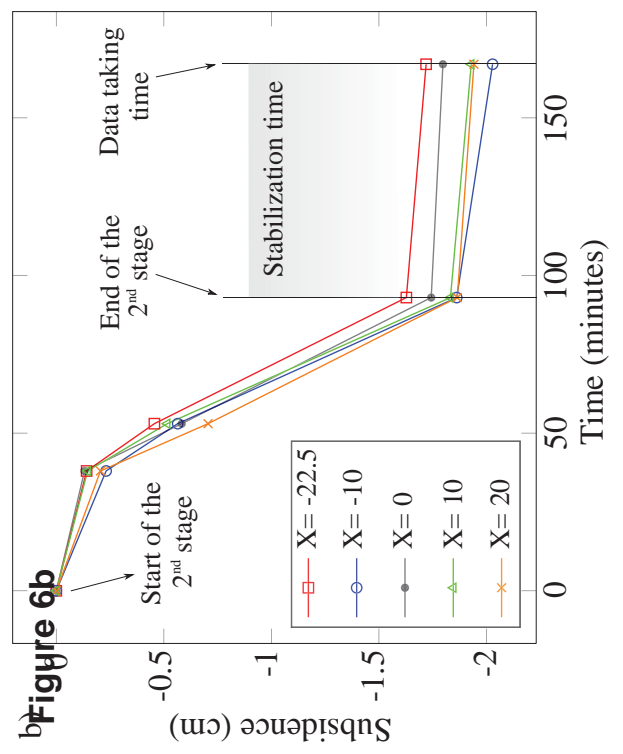




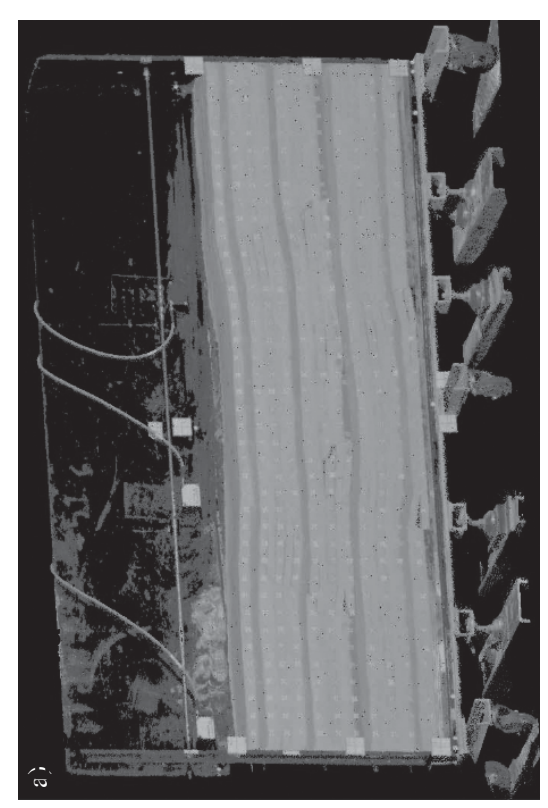




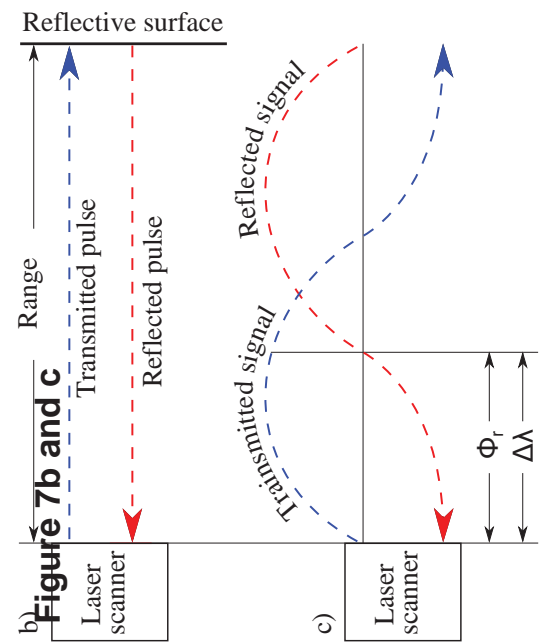




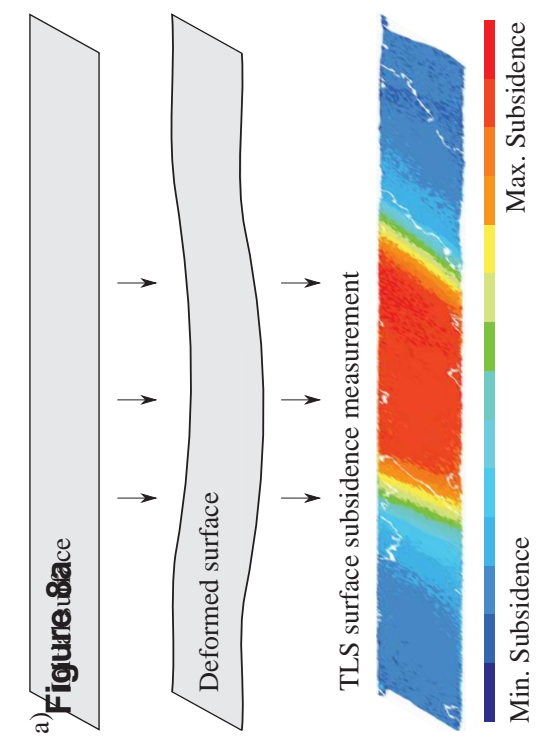




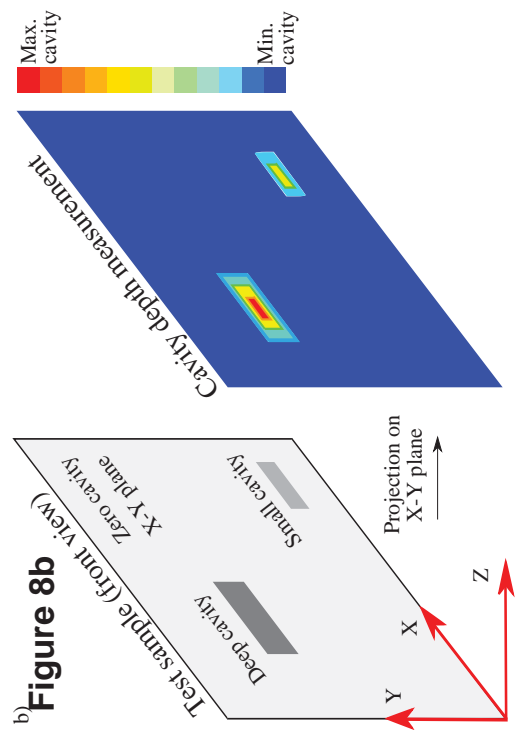




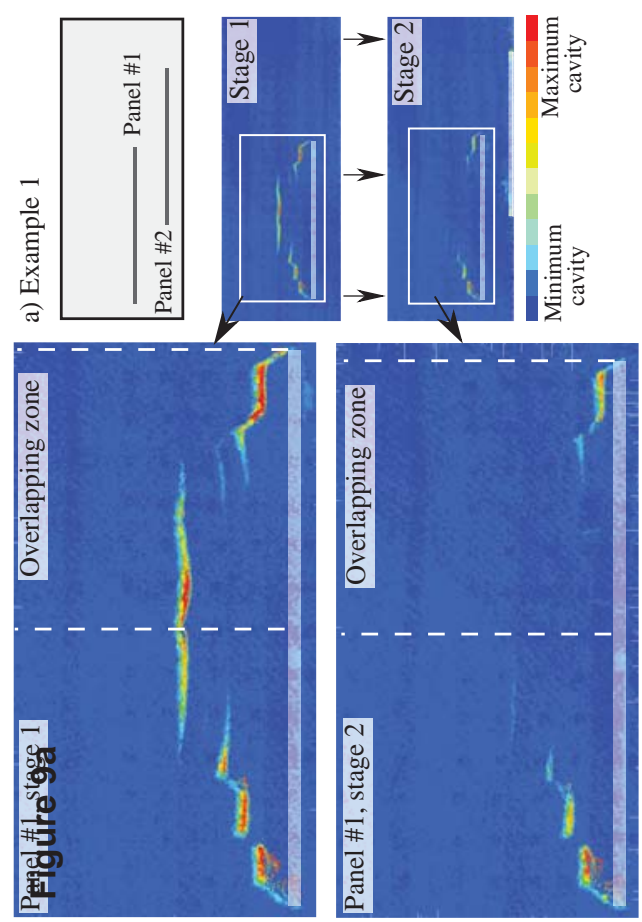




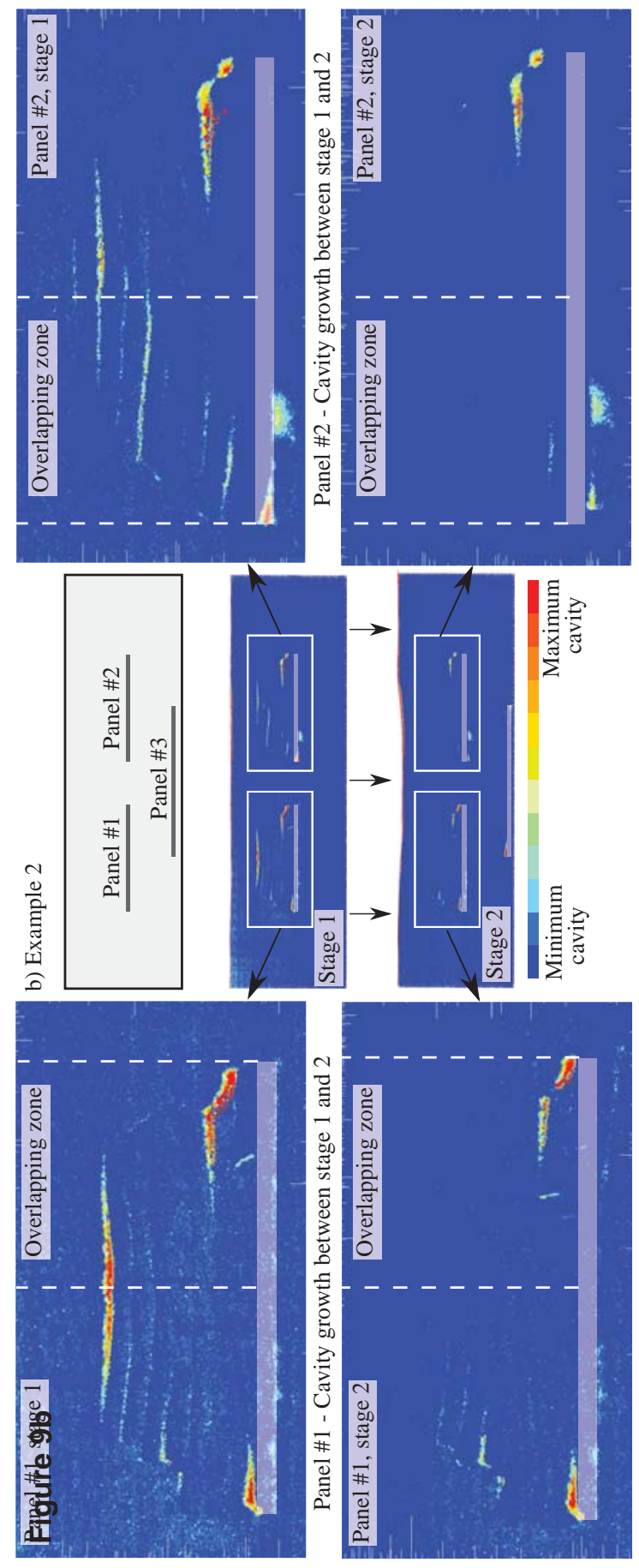




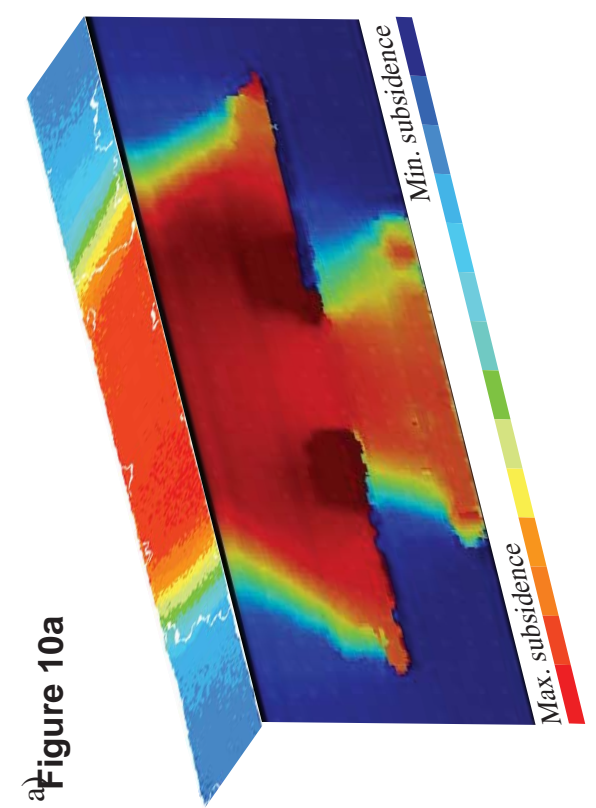




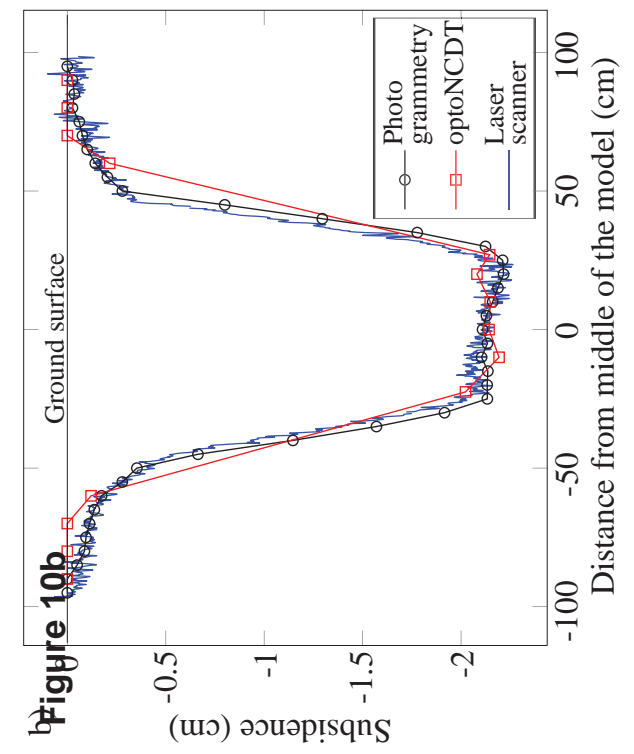




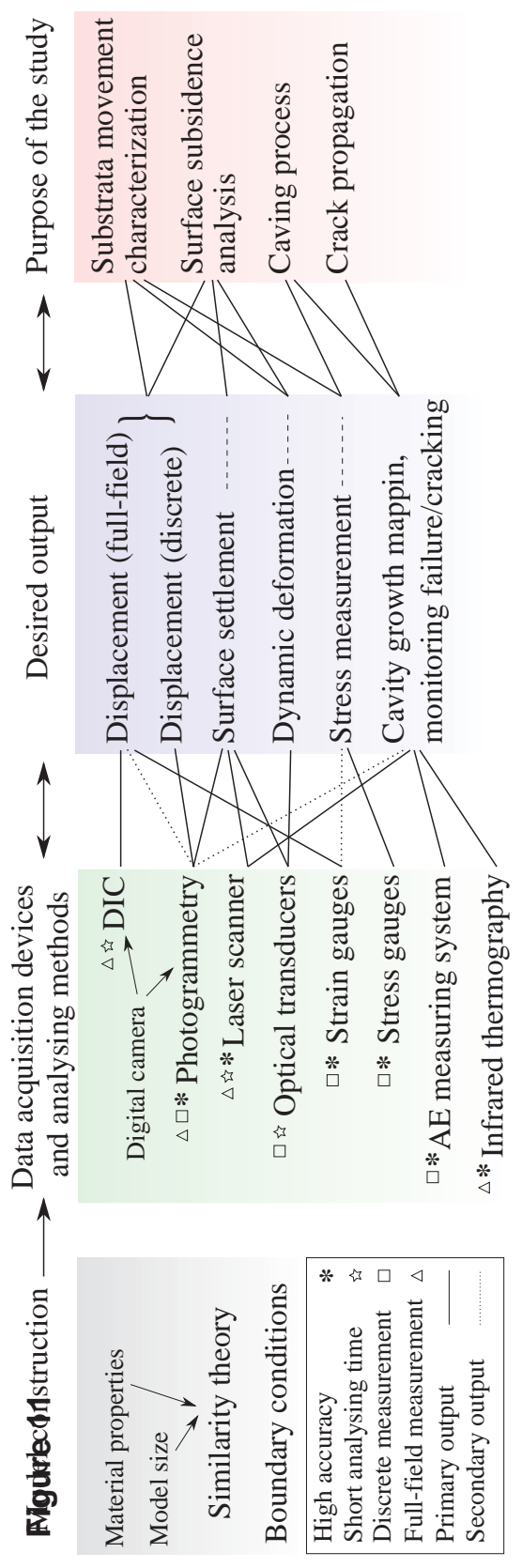




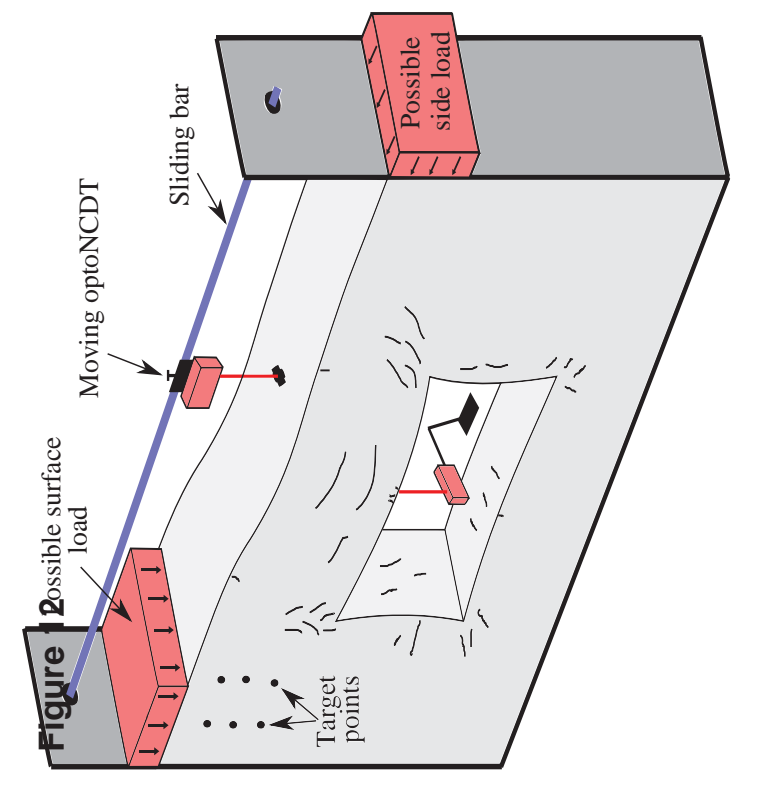

